\title{
Evaluation of US Food and Drug Administration- recommended abuse-potential questions in chronic pain patients without history of recreational opioid use: results and plan for research
}

This article was published in the following Dove Press journal: Journal of Pain Research

\author{
Joseph A Boscarino' \\ Carrie A Withey' \\ Ryan J Dugan' \\ Thomas Alfieri \\ 'Department of Epidemiology and \\ Health Services Research, Geisinger \\ Clinic, Danville, PA 17822, USA; \\ 2Medical Affairs, Purdue Pharma, \\ Stamford, CT 0690I, USA
}

Background: Existing patient-reported outcome (PRO) assessments that measure the human abuse potential for opioid analgesics have been tested exclusively in experienced recreational opioid users, as required by US Food and Drug Administration (FDA) guidance.

Methods: The goals of the current studies were to modify items from FDA-recommended abuse potential PRO assessments to specify the analgesic benefits versus the euphoric effects of opioids and to ascertain the clarity, understandability, appropriateness, and validity of the modified questions. This was achieved by conducting cognitive debriefing interviews (CDIs) with patients ( $\geq 18$ and $<65$ years) who have chronic pain, were prescribed an opioid daily dose of at least 80 morphine-equivalent milligrams ( $>30$ days to $\leq 180$ days from the date of interview), and did not have a history of recreational opioid use.

Results: Participants in study $1(n=30)$ and study $2(n=7)$ had a better understanding of the items designed to measure the concepts of drug liking and items designed to measure the desire to take a drug again when reasons for liking and desire to take again were included in the item wording (namely, "due to pain relief" and "excluding pain relief"). Most participants indicated no interest in taking their medication for reasons other than pain relief.

Conclusion: Modification of questions in the PRO assessment improved patient understanding of "drug liking" and "desire to take again." Patients with chronic pain who were not recreational opioid users understood the difference between the analgesic and euphoric effects of an opioid drug. The modified questions should assist future researchers in providing a more accurate assessment of the abuse potential of an opioid, as required by regulatory agencies.

Keywords: opioid analgesics, patient-reported outcomes, chronic pain, assessments, substancerelated disorders, United States Food and Drug Administration, abuse potential

\section{Introduction}

During the safety evaluation of a new drug in development for approval, the Food and Drug Administration (FDA)'s “Assessment of abuse potential of drugs: guidance for industry" requires that the abuse potential of a drug be assessed in subjects who are experienced recreational users and who are able to ". . . demonstrate a meaningfully different response from that produced by placebo". ${ }^{1}$ To date, patient-reported outcome (PRO) assessments that measure the human abuse potential for opioid analgesics (including the Drug Effects Questionnaire [DEQ]) have been tested primarily in
Correspondence: Joseph A Boscarino Department of Epidemiology and Health Services Research, Geisinger Clinic, 100 North Academy Avenue, MC 44-00 Danville, PA 17822, USA

Tel +I 5702149622

Fax + I 570214945 I

Email jaboscarino@geisinger.edu 
recreational opioid users, as required by the FDA guidance. ${ }^{1}$ Measuring the abuse potential of opioids in recreational users provides useful information; however, opioid analgesics are approved by the FDA for the treatment of pain and are not intended for recreational drug use. Therefore, accurately assessing abuse potential in nonrecreational users is important, but may be difficult when using PRO assessments developed for use with recreational users. Given the current high rates of opioid-use problems and opioid overdoses reported in health care delivery systems, ${ }^{2-4}$ accurately assessing abuse potential in nonrecreational users is of the utmost importance.

In addition to the DEQ, commonly used measures in assessing opioid-abuse potential in clinical studies include:

- the $100 \mathrm{~mm}$ Drug-Liking Visual Analogue Scale (DL-VAS) ${ }^{5}$

- the $100 \mathrm{~mm}$ Overall DL-VAS (ODL-VAS) ${ }^{5}$

- the Severity of Dependence Scale (SDS) ${ }^{6}$

- the Drug Abuse Screening Test (DAST10). ${ }^{7}$

The DL-VAS and ODL-VAS are qualitative scales recommended to assess opioid-abuse potential in clinical trials focused on reports of drug liking and feeling high. ${ }^{5}$ The SDS is a quantitative scale focused on addiction potential that has been extensively used in the drug-abuse field across different classes of psychoactive substances. ${ }^{6}$ The DAST10 is a brief ten-item drug-abuse screener designed to assess drug abuse and recreational drug misuse. ${ }^{7}$ While these instruments have been successfully used with recreational drug users, they may cause confusion and produce misleading results when administered to patients taking opioid analgesics for pain management without clarifying contextual information.

For example, current VAS items may confuse the euphoric effects of the opioid with the analgesic benefits of the medicine. ${ }^{8}$ These scales may not be able to distinguish a patient's liking a drug for pleasure from liking a drug for pain relief. Patients with chronic pain who experience poor pain management often exhibit behaviors consistent with addictive behaviors that can further confound PRO assessments, such as a focus on drug seeking, requesting medication prior to scheduled dosing, requesting specific opioids, and anxiety about future medications. ${ }^{8}$ Therefore, legitimate requests for opioids with the distinct purpose of relieving chronic pain can be confused with drug-seeking behavior for abuse.

There is an important and unmet need for proper validation of these PRO instruments in a population of patients diagnosed with chronic pain who do not have a history of opioid recreational use. Modification of any unclear scales or items regarding opioid-abuse potential in clinical trials and observational studies may help to determine more accurately the reasons for a patient endorsing liking of an opioid and wanting to take the drug again, particularly whether the opioid is being used for pain relief, or for reasons other than pain relief. The goals of the current two studies were to assess if PRO assessments currently administered to recreational drug users can be appropriately understood and interpreted by pain patients, and if new VAS scale items would be understood by pain patients. Two sets of cognitive debriefing interviews (CDIs) with patients who had chronic pain and were not recreational opioid users were conducted. CDIs are often used in exploratory survey research to assess and improve the accuracy of survey questions. ${ }^{9-11}$ Patients evaluated standard abuse-potential PRO assessments and additional VAS items that were designed to distinguish the analgesic benefits vs the euphoric effects of the opioids to ascertain the clarity, understandability, appropriateness, and validity of these scales and modified items in patients taking opioid analgesics for pain management, consistent with debriefing methods commonly used in survey research. ${ }^{9}$

\section{Methods}

\section{Study participants}

Eligibility requirements for both studies were as follows:

\section{Inclusion criteria}

1. current clinical diagnosis of chronic pain

2. at least 18 years of age and $<65$ years of age

3. prescribed extended-release oxycodone or hydrocodone or immediate-release oxycodone or hydrocodone (allowing for combination products containing acetaminophen, and allowing for concomitant use of over-the-counter nonsteroidal anti-inflammatory drugs and paracetamol)

4. minimum daily prescription dose of 80 morphine-equivalent $\mathrm{mg}$

5. prescription period $>30$ days to $\leq 180$ days from the date of interview, before day of CDI.

\section{Exclusion criteria}

1. any history of opioid abuse or physician-suspected opioid abuse, including recreational opioid use

2. use of more than one opioid product or an opioid other than hydrocodone or oxycodone

3. patients taking pregabalin or gabapentin

4. inability to communicate using spoken English or inability to read the English Language

5. unwillingness to participate in a confidential interview or any other characteristic that prohibited the person from 
understanding the informed consent form and/or completing the interview, determined by either the recruiter or the interviewer.

\section{Interviews}

The CDIs, conducted by experienced qualitative researchers, began with a brief discussion of participants' histories with chronic pain and opioid medications, and probed for the concepts that patients with chronic pain feel are important regarding the potential abuse of prescription opioids. Consistently with CDI methods, interviewers followed a structured interview guide, which detailed the flow and content of the conversation for the individual interviews. This methodology is commonly used in PRO research. ${ }^{12}$

Before administering the instruments, interviewers explained to study participants that the assessments were designed to be asked of a person who is enrolled in a clinical trial, ie, these questions would normally be asked shortly after a person took the drug under investigation. Participants completed the PRO assessments as outlined herein with no interference or instruction from the interviewer, and then were queried to determine the clarity and relevance of the items.

The CDIs were one-on-one, audio-recorded, and conducted at a research center. Written informed consent was obtained for all participants prior to the study procedures. The New England Independent Review Board (Newton, MA) served as the institutional review broad for this study, and approved the protocol, informed consent form, and all written information provided to participants.

\section{PRO assessments included in CDIs}

All participants in study 1 completed the $100 \mathrm{~mm}$ DL-VAS, original and new items, ${ }^{5}$ and the ODL-VAS, original and new items. ${ }^{5}$ All participants in study 2 completed the 100 mm DL-VAS, original and new items, ${ }^{5} 100 \mathrm{~mm}$ ODL-VAS, original and new items, ${ }^{5} \mathrm{SDS},{ }^{6}$ and DAST $10 .{ }^{7}$

\section{New VAS items}

The original DL-VAS and ODL-VAS were augmented with six new items. The VAS item "I would take this drug again" was used as a model to create the following new items:

1. "I would take this drug again for pain relief"

2. "Excluding pain relief, I would take this drug again."

The response option used for the original item, a $100 \mathrm{~mm}$ scale ranging from "Definitely not" to "Definitely so," was unaltered. Similarly, the items "At this moment, my liking for this drug is?" and "Overall, my liking for this drug is?" were used as models to create the following new items:

1. "At this moment, my liking for this drug due to pain relief is?"

2. "At this moment, excluding pain relief, my liking for this drug is?"

3. "Overall, my liking for this drug due to pain relief is?"

4. "Overall, excluding pain relief, my liking for this drug is?"

The response option for these four items remained a 100 $\mathrm{mm}$ scale ranging from "strong disliking" at the $0 \mathrm{~mm}$ mark to "neither like nor dislike" at the $50 \mathrm{~mm}$ mark to "strong liking" at the $100 \mathrm{~mm}$ mark.

\section{Qualitative analysis}

Qualitative analysis was used to recognize common participant terminology, determine how participants interpreted the items, and ascertain how participants considered their answers to such questions. Interview transcripts were reviewed and coded by a specialist. Terms and phrases were captured for classification into similar domains or concepts. Researchers analyzed responses to evaluate the clarity, understandability, appropriateness, and validity of the original and newly created items within this patient population. Interviews lasted up to 1 hour, 15 minutes of total discussion time.

\section{Results \\ Study I}

Thirty participants met the study-inclusion criteria and completed the CDIs. The majority of participants were women $(n=22,73.3 \%)$ and African-American $(n=26,86.7 \%)$. The mean age of participants was 46 years and the average time prescribed an opioid was 88 days. The interviews were conducted at a research facility in Snellville, GA.

\section{Items assessing desire to take the drug again}

The original version of this item ("I would take this drug again") was well understood by 27 participants, and the remaining three participants indicated that they were not sure how to respond. The inclusion of the phrase "for pain relief" made this item clear to all 30 participants, none of whom expressed concerns about how to respond. When presented the item "Excluding pain relief, I would take this drug again," 29 of the 30 participants spontaneously said they would take an opioid only for pain relief. Participants understood that some people took the medicines to "get high," but that they personally had no reason to take an opioid other than pain relief. 
When responding to these new items, most participants placed marks on opposite sides of the $100 \mathrm{~mm}$ VAS, indicating that the different items elicited different responses. A total of 26 participants indicated a higher likelihood to take the drug again due to pain compared to taking the drug again for reasons other than pain, and no participant indicated a higher likelihood to take the drug again for reasons other than pain compared to for pain relief. The remaining four participants gave similar responses to the two items (ie, the different item wordings did not impact their response). Table 1 lists the participant responses.

\section{Items assessing drug liking}

Participants were asked to explain what was meant by "due to pain relief" and "excluding pain relief" when added to the original items "Overall, my liking for this drug is," and "At this moment, my liking for this drug is." Participants were able to distinguish the difference between "due to pain relief" (taking the medicine as a prescribed drug because one is in pain) and "excluding pain relief" (treating the medicine as a "narcotic" and taking it "to get high"). Participant responses indicated that they had better understanding of the "drug liking" items when "due to pain relief" was added. Thirteen participants indicated that the original "liking" items were unclear. However, with the addition of "due to pain relief," only one participant found these items still to be unclear.

\section{Item assessing "feeling high"}

Of 30 participants, 29 were able to provide a definition, interpretation, or synonym for the phrase "I am feeling high." The most common responses were "euphoric," "lightheaded," "dizzy," "and drowsy." A few participants compared the "high" feeling to being drunk or to the use of marijuana.
Participants indicated that the statement "I am feeling high" is clearly understood, and none of the 30 participants had difficulty in responding to this item.

\section{Study 2}

Seven participants met the study-inclusion criteria and completed the CDIs. There were five women (71\%) and two men (28\%). Participants were Caucasian $(n=4,57 \%)$, Hispanic $(n=2,28 \%)$, and African-American $(n=1,15 \%)$. The mean age of participants was 45.2 years, and average time on opioids was 122 days. Interviews were conducted in Los Angeles, CA and Chicago, IL.

\section{DL-VAS and ODL-VAS}

Similarly to study 1 , the VAS was generally intuitive and easy for participants to use. The new item "I would take this drug again for pain relief" was described as "straightforward," easy to comprehend, and respond to. Participants were also able to clearly interpret the more complex items containing the "excluding" phrase, such as "Excluding pain relief, I would take this drug again."

Also similarly to study 1 , participants' response patterns indicated that they made a distinction between the two new variations of each original item, and the direction of the responses was in an expected manner. Namely, participants' responses to "Overall, my liking for this drug due to pain relief is" was higher (ie, more likable) compared to "Overall, excluding pain relief, my liking for this drug is."

\section{SDS}

Overall, participants felt confident responding to the SDS items. However, several issues were identified that led some participants to feel somewhat confused or unsure about their meaning.

Table I Select statements from cognitive debriefing interviews

\begin{tabular}{|l|}
\hline Participant statements \\
\hline In response to items assessing desire to take drug again \\
- "Well, I mean I would take it again definitely for pain reasons and just pretty much pain reasons." \\
- "I it wasn't for the pain relief, I would not be taking it." \\
- "Would I take it only for pain relief if it had no attachment of euphoric feeling? Yes, I would." \\
\hline In response to items assessing likability of drug \\
- "Now, this one says: 'At this moment, excluding pain relief, my liking for the drug is ...", and I put 'neither like nor dislike, because, really, for \\
- "This one I can say clearly is that it's a matter of if I'm not taking it for pain, I have no dislike or like for it." \\
- "I'm trying to think: the 'like' or the 'not like"? Sometimes you take stuff whether you like it or not." \\
\hline
\end{tabular}


Item I: Do you think your use of drug $X$ was out of control?

Participants believed that being out of control meant they were unable to or unwilling to use the medication as intended and lacked the control to change their behavior. Participants questioned why they would be asked to consider being out of control for a medication they were using according to their prescriptions.

Item 2: Did the prospect of missing a fix (or dose) make you anxious or worried?

The word "fix" was both confusing and disturbing to some participants. "Fix" is strongly associated with illicit drugs, whereas "dose" indicates medication. Because they were responding about their use of pain medication, participants suggested the word "fix" be removed. A couple of participants were somewhat offended by the apparent implication that they were using illegal drugs, rather than taking medication. One participant completed the questions with the frame of reference that she was being asked about illegal drug use, rather than the pain medication she was relying on.

\section{Item 3: Did you worry about your use of drug $X$ ?}

This item was easy to read and understand, although some participants questioned why they would worry about their pain medication, as they were following the instruction of their prescribing doctor.

\section{Item 4: Did you wish you could stop?}

There was confusion among participants about the intent of this item. One interpretation was that the item was referring to an inability to stop taking the medication because the subject had become dependent on the medication beyond that needed to manage the pain. This interpretation was somewhat bothersome for those who had already concluded the items were not interested in their use of legal, prescription medication. A second interpretation was that the item may have been referring to the ability to stop taking the pain medication because the participant no longer experienced pain. With this second interpretation, participants considered responding in the affirmative.

Item 5: How difficult did you find it to stop or go without drug $X$ ?

As participants were still taking their medication, they believed that part of the question (about stopping) was irrelevant. Participants considered how difficult they thought it might be for them to go without their medication, given their current pain.

\section{DASTIO}

This instrument was considered easier and more natural for participants to complete. Several commented that the questions were "natural" and "straightforward." Even so, a few items presented a few challenges for participants.

\section{Item 2: Do you abuse more than one drug at a time?}

Participants did not believe they were abusing any drug; including the pain medication they were currently using. The implication that participants were abusing their medication was somewhat offensive in general. Furthermore, a negative response did not accurately portray the fact that they were not abusing any drugs, just that they were not abusing more than one.

Item 3:Are you always able to stop using drugs when you want to?

The composition of this item was awkward. Participants were hesitant to stop taking their medication because of their pain.

The remaining items were understood by participants.

\section{Discussion}

These qualitative studies in patients with chronic pain have several meaningful findings, with implications for future studies intending to measure opioid-abuse rates and opioid clinical trials. Using CDIs, the study results showed that PRO assessments previously validated for measurement of opioid-abuse potential in recreational drug users as per the FDA guidance ${ }^{1}$ should be modified minimally to elicit more accurate and well-defined responses in a nonrecreational opioid-use population of patients with chronic pain. The results of this study provide evidence that the addition of such clauses as "due to pain relief" and "excluding pain relief" are important and necessary for any evaluation of opioid "drug liking" and the desire to take the drug again. Participants in the current studies responded consistently that the inclusion of these new items on the VAS provided greater clarity and improved understanding of the concepts of liking and desire to take again compared to the existing items. Furthermore, items that attempt to measure a respondent's desire to stop using a drug should be carefully considered when applied to pain patients, as respondents may not clearly separate 
their desire to stop using a medicine with their desire to stop experiencing pain.

Participants also clearly understood the term "feeling high." Given this understanding of the euphoric effects of opioids, these participants still consistently responded that they would take an opioid only for pain and not for other reasons. In an analysis of DEQ items from three placebocontrolled studies, "like" was associated with the euphoric and mood-altering effects of a substance of abuse (amphetamine, nicotine, or alcohol). ${ }^{13}$

Participants in this study were screened to exclude those with any history of opioid abuse or physician-suspected opioid abuse, including recreational opioid use. While the number of subjects meeting the inclusion criteria and included in this study was fairly small, in this population, even after taking a relatively high dose of opioids for an extended period of time, they were not diagnosed with an opioid-use disorder. In the current health care environment, however, clinicians must balance the potential benefits of long-term prescription-opioid therapy with the serious risks for opioid-use disorder, abuse, drug overdose, and increased drug-related mortality. ${ }^{2-4}$ In a recent editorial, Gorfinkel et al suggested that chronic pain patients may be misdiagnosed because the characteristics of mismanaged pain may mimic the criteria of opioid-use disorder. ${ }^{14}$ The need to understand clearly the reasons behind "liking" an opioid and the desire to "take it again" seem of paramount importance in patient populations. ${ }^{15}$

The results of the current study should be considered when designing future studies that evaluate the abuse potential of opioid analgesic drugs in patients with chronic pain who are nonrecreational opioid users. The current study presents qualitative results, and while participants were screened to meet specified criteria, the study design did not include randomization, and thus represents an observation study. The majority of participants in study 1 were AfricanAmerican women, reflecting the population from which they were drawn. Participants in study 2 represented a more even balance of sex and ethnicity. Quantitative studies need to be conducted to support the conclusions of this CDI research. The new items in the VAS should be further tested to confirm that they satisfy the unmet need to distinguish between the pain-relieving effects and the nontherapeutic, euphoric, or hedonic effects of an opioid. Researchers should carefully examine all PRO instruments originally designed for experienced recreational opioid users when applied to patients with chronic pain to avoid misidentification or misclassification of patients. Based on this research, a quantitative study of abuse potential is currently being planned among a broader cross-sectional sample of pain patients that includes recreational, nonrecreational, short-term, and long-term users of opioid medications. Traditional and modified versions of the PROs discussed in this article will be used in future research.

\section{Conclusion}

This study showed that modification of questions in PRO assessments improved patient understanding of the concepts "drug liking" and "desire to take again." This study appropriately targeted the population for which the drug was intended (ie, patients with chronic pain with no history of recreational drug use). By comparison, the SDS presented conceptual issues for these nonrecreational opioid-use patients, because this scale was developed for and normed on substance-abusing populations. The results of this study suggest that these patients understand the difference between the analgesic and euphoric effects of an opioid drug, and that the modified assessments will assist researchers in providing a more accurate assessment of the abuse potential of an opioid, as required by regulatory agencies.

\section{Author contributions}

All authors contributed to data analysis, drafting or revising the article, gave final approval of the version to be published, and agree to be accountable for all aspects of the work.

\section{Acknowledgments}

The authors would like to thank Kim Poinsett-Holmes (Evidera, Bethesda, MD) for medical writing/editorial assistance during the development of the manuscript. This research and editorial support for the development of the manuscript was funded by Purdue Pharma, LP.

\section{Disclosure}

JAB, CAW, and RJD are employees of Geisinger, Danville, PA. Geisinger collaborated with and received payment from Purdue Pharma for this study. TA is a salaried employee of Purdue Pharma. The authors report no other conflicts of interest in this work.

\section{References}

1. Department of Health and Human Services (DHSS), Food and Drug Administration, Center for Drug Evaluation and Research. Assessment of abuse potential of drugs. Guidance for Industry. 2017:1-34.

2. Maeng DD, Han JJ, Fitzpatrick MH, Boscarino JA. Patterns of health care utilization and cost before and after opioid overdose: findings from 10-year longitudinal health plan claims data. Subst Abuse Rehabil. 2017;8:57-67.

3. Boscarino JA, Kirchner HL, Pitcavage JM, et al. Factors associated with opioid overdose: a 10-year retrospective study of patients in a large integrated health care system. Subst Abuse Rehabil. 2016;7: 131-141. 
4. Boscarino JA, Rukstalis M, Hoffman SN, et al. Risk factors for drug dependence among out-patients on opioid therapy in a large US healthcare system. Addiction. 2010;105(10):1776-1782.

5. Setnik B, Roland CL, Pixton G, Webster L. Measurement of drug liking in abuse potential studies: a comparison of unipolar and bipolar visual analog scales. J Clin Pharmacol. 2017;57(2):266-274.

6. Gossop M, Darke S, Griffiths P, et al. The Severity of Dependence Scale (SDS): psychometric properties of the SDS in English and Australian samples of heroin, cocaine and amphetamine users. Addiction 1995;90(5):607-614.

7. Skinner HA. The drug abuse screening test. Addict Behav. 1982;7(4): 363-371.

8. Greene MS, Chambers RA. Pseudoaddiction: Fact or fiction? An investigation of the medical literature. Curr Addict Rep. 2015;2(4): 310-317.

9. Groves RM, Fowler FJ, Couper MP, Lepkowski JM, Singer E, Tourangeau R. Survey Methodology, 2nd Edition. New York: NY: Wiley; 2009:259-290.
10. Ploughman M, Austin M, Stefanelli M, Godwin M. Applying cognitive debriefing to pre-test patient-reported outcomes in older people with multiple sclerosis. Qual Life Res. 2010;19(4):483-487.

11. Remor E, Fuster MJ, Ballester-Arnal R, et al. Development of a new instrument for the assessment of psychological predictors of well-being and quality of life in people with HIV or AIDS. AIDS Behav. 2012;16(8): 2414-2423.

12. Stokes J, Evans CJ, Pompilus F, Shields AL, Summers KH. Development of a questionnaire to assess the impact of chronic low back pain for use in regulated clinical trials. Patient. 2013;6(4):291-305.

13. Morean ME, de Wit H, King AC, Sofuoglu M, Rueger SY, O’Malley SS. The drug effects questionnaire: psychometric support across three drug types. Psychopharmacology (Berl). 2013;227(1):177-192.

14. Gorfinkel L, Voon P, Wood E, Klimas J. Diagnosing opioid addiction in people with chronic pain. BMJ. 2018;362:k3949.

15. Fischer MA, McKinlay JB, Katz JN, et al. Physician assessments of drug seeking behavior: A mixed methods study. PLoS One. 2017;12(6): e0178690

\section{Journal of Pain Research}

\section{Publish your work in this journal}

The Journal of Pain Research is an international, peer reviewed, open access, online journal that welcomes laboratory and clinical findings in the fields of pain research and the prevention and management of pain. Original research, reviews, symposium reports, hypothesis formation and commentaries are all considered for publication.

\section{Dovepress}

The manuscript management system is completely online and includes a very quick and fair peer-review system, which is all easy to use. Visit http://www.dovepress.com/testimonials.php to read real quotes from published authors. 